\title{
Philosophical and Methodological Aspects of a Mixed-Methods Research: a Review of the Academic Literature
}

\author{
Dr. Salman Bashir ${ }^{1}$ \\ Dr. Sumaiya Syed ${ }^{2}$ \\ Dr Jawaid Ahmed Qureshi ${ }^{3}$
}

\begin{abstract}
The selection of an appropriate research method is extremely critical for a value added research. The purpose of this study is to critically review the academic literature of a mixed-methods research and to examine the philosophical and methodological aspects that rationalize how and why a mixed-methods research could be useful in a management study. Hence, this paper critically reviews the 'nuts and bolts' of a 'mixed methods' research grounded on the research question intentionally drawn for illustration. In terms of contribution, this paper contributes to literature on a 'mixed methods' literature by providing a holistic view of a research design that manage the both quantitative and qualitative threads during the same phase of the research process typically termed as concurrent design in 'mixed methods' research. This particular design entail researchers to implement, upheld each thread independently during analysis, and finally mixes the results conjointly in such a way that they portray the apparent picture of the underlying phenomenon.
\end{abstract}

Keywords: Mixed-Methods Research, Mixed-Methods Validity, Research Design, Convergent Parallel Design

\section{INTRODUCTION}

The selection of the research method is not subject to the merits and demerits of that method. Likewise, there is no such yardstick that could corroborate the suitability of any of the research methods under predisposed circumstances. It has been confirmed from the literature that the research design may be determined by the nature of the research problem and the way through which it seeks answers (Crotty, 1998). The researchers usually employ mixed-methods to unfold complex research topics. However, the balanced philosophical stance plus the potential challenges during data collection also contemplated during the process of methodology selection. For mixed-method research, Johnson et al. wrote that:

...the type of research in which a researcher or team of researchers combines elements of qualitative and quantitative research approaches (e.g. use of qualitative and quantitative viewpoints, data collection, analysis, inference techniques) for the broad purposes of breadth and depth of understanding and corroboration (Johnson et al., 2007, p.113).

\footnotetext{
1 - Assistant Professor at Department of Business Administration, Shah Abdul Latif University, Khairpur.

Email: salman.memon@salu.edu.pk

2 - Assistant Professor at Department of Business Administration, Shah Abdul Latif University, Khairpur.

3 - Associate Professor at Shaheed Zulifqar Ali Bhutto Institute of Science \& Technology (SZABIST) Karachi.

JISR-MSSE

Volume 1

Number 15

Jan-June 2017 
In recent years, the mixed-methods has become the third paradigm (Johnson and Onwuegbuzie, 2004). In case of management studies where positivism has long subjugated, a mixed-methods is now widely accepted in a wide variety of disciplines such as human resources, marketing, organisational behaviours, strategic management, knowledge management and human psychology (Grafton et al., 2011; Jogulu and Pansiri, 2011). Therefore, the pristine use of inductive and deductive logic in a single study can improve the results so that researchers can make inferences with more confidence. The inductive and deductive logic of enquiry permits researchers to uniformly embark on theory generation and hypothesis testing without conciliating one for the other. Likewise, identical divisions between two methods with the help of multiple data sources and analysis firmly create contrary views and findings. For that purpose, the researcher has employed mixed-methods for plunging over-dependence on quantitative data to define the subjective nature of the social phenomena and experiences. In a more abstract sense, the mixed-methods approach addresses several critical issues concern with theoretical and methodological practices. It impacts upon the study's aims and outcomes as it provides a reasonable agreement between theories with methodology. It also ensures the reliability and validity of the overall research findings. In addition, the conservative ascendancy of any one particular research technique, either qualitative or quantitative dichotomies, creates research outcomes of high standing (Jogulu and Pansiri, 2011). In general, validity and reliability have primarily resulted from quantitative research. However, a qualitative study offers significant aspects in the course of the subjective interpretations of experiences that provide conceivable answers in relation to social phenomena and individual experiences (Pansiri, 2005; Jogulu and Pansiri, 2011).

Generally speaking, no single research methodology is inherently superior to any other methodology (Kaplan and Duchon, 1988). For example, in the late 1980s and early 1990s, social science researchers utilized elements of both the positivist and post-positivist approaches in a single study in order to improve the value of research. Therefore, the choice of mixed-methods research was not due to the merits and demerits of different substitutes, but the nature of the research problem. In a management studies, mixed-method as a methodology have been generally accepted (Bryman, 2005). Specifically, it was literally widespread in human resource management and knowledge management research literature (Modell, 2010 and Cassell et al., 2006).

\section{Research Question Design in a Mixed-Methods Research}

The way of writing a research questions in a mixed-methods design is generally recommended by distinguished methodologists (e.g. Bryman, 2007, Creswell and Plano Clark, 2007, Tashakkori and Creswell, 2007). Although, the literature has revealed no legitimate source that can prescribe how to write a mixed-methods research question because it is somewhat complex to outline a research question in a mixed-methods study than any particular method study. According to Onwuegbuzie and Leech (2006), a mixed-methods research questions are questions that entrench both qualitative and quantitative research questions within the individual question. It requires both qualitative and quantitative data to be collected and analyzed either sequentially, concurrently, or iteratively. Specifically, mixed-methods research questions can be stated by one of three ways (Tashakkori and Creswell, 2007). 
The first way is to write qualitative and quantitative questions separately followed by an open mixed-methods question. The second way is to write a combined research question which is further divided into separate qualitative and quantitative sub-questions. Likewise, the third way is to write research questions of each phase as the study progresses. Generally speaking, the way of writing a mixed-methods question is someway different individual methods that predominantly consolidates the objective of the study. The main research question is divided into two sub questions in such a way that the both sub questions (i.e. qualitative and quantitative) addresses underlying objectives of that method. For example, the mixed-methods research question can be outlined as follows:

\section{Mixed-Methods RQ: How does organisational culture affect knowledge creation process?}

Afterwards, the mixed research question can be divided into separate qualitative and quantitative sub-questions that are answered in each strand of the study. For example, the quantitative sub-question is outlined as follows:

\section{Quantitative RQ: What may be the relationship between organisational culture and knowledge creation process?}

In terms of methods, the first sub-question can be answered quantitatively during hypothesis testing (or path analysis) through the model fit using structural equation modelling (SEM). The quantitative strand intends to investigate the structural relationship between organisational culture and the knowledge creation process. In case of this example, the qualitative question can be outlined in order to unfold the core issues related to the policy framework of Pakistani commercial banks from knowledge culture and knowledge management implementation and use. The qualitative sub-question within a mixed-methods research question can outline in this way:

Qualitative RQ: How does the senior management promote knowledge creation and sharing culture? Precisely, the senior leadership is the core partaker in formulating policies and setting organisational goals and objectives. They are also accountable to provide sustainable organisational culture that may be conducive in creating and managing new employee knowledge. Hence, qualitative data from the purposely selected participants is expected to provide a more comprehensive picture of the underlying problems more explicitly. Therefore, in the qualitative strand, senior leadership and HR heads who are involved in the policymaking can be targeted for interviews. Specifically, qualitative information is valuable for investigating social, organisational or cultural upbringing of underlying phenomenon and unfolding people to accept wisdom towards any issue or problem (Corbin and Strauss, 2008). Thus, the purpose of qualitative information was to probe quantitative results in more depth and to seek opinions and perceptions of the target population because interviewing is one of the important tools of data collection familiar with participant perception, belief and behavior (Guthrie, 2010; Creswell, 2013).

Generally speaking, quantitative and qualitative sub-questions are required to be answered either quantitatively or qualitatively. Therefore, mixed-methods allow researchers to acquire data about situations, behavioral patterns, norms or practices at one point of time. The quantitative component allows the researchers to examine more variables about the real world phenomenon that is typically not possible with other approaches. For this, a questionnaire survey can be appropriate for quantitative analysis in order to draw inferences from this data

\begin{tabular}{llll}
\hline JISR-MSSE & Volume 1 & Number 15 & Jan-June 2017 \\
\hline
\end{tabular}


regarding existing relationships. In contrast, the qualitative component is primarily concerned with answering 'why' and 'how' questions within the context. For this, the interview survey intends to examine the behavioral pattern and specific phenomena in more detail. However, the dialogue between quantitative and qualitative components normally commenced at the opening of the research design. For example, "a valid mixed methods research is much like a dialogue or debate, and the idea is then to construct a negotiated account of what they mean together" (Bryman, 2007, p.21).

\section{Philosophical Aspects of a Mixed-Methods Research}

Generally, in research, a choice of research designs may be dependent on two aspects (Denzin and Lincoln, 2011). First is the research aim and objectives; whereas, the second is the researcher's philosophical understanding, experience, and personal beliefs or assumption. Although, this is not as simplistic as it appears. For example, the final decision of research methods is not just a matter of random choice from the set of available methods such as interview, questionnaire, focus group and observation. Also, the choice of research methodology is always susceptible to the philosophical assumptions of the researcher because philosophical perspective not only explains the nature of society, but it also unveils the nature of science through which new knowledge can be produced (Burrell and Morgan, 1979).

More specifically, the researcher's philosophical assumptions about ontology, epistemology and human nature play an important role in the selection of research methodology (Gill and Johnson, 2002). Thus, after deciding ontological and epistemological assumptions and taking an objective-subjective versus positive-phenomenological position of the research, an important decision related to methodology needs to be taken. In other words, an understanding of philosophical issues can be noteworthy due to numerous reasons. For example, different paradigms lead to studying underlying phenomena in different ways. It not only describes a number of organisational phenomena from different perspectives, but also highlights different kinds of knowledge that derive throughout observing the same phenomena from different philosophical perspectives (Hatch, 2012).

\section{Issues of Ontological Assumptions}

Ontological assumptions may either be extremely objective or extremely subjective. An objectivist asserts that social reality is independent of social actors. It is an objective phenomenon that provides accurate observation and measurement (Morgan and Smircich, 1980). In contrast, 'subjectivists' negate the 'objectivists' view. According to the Morgan and Smircich (1980, p.494) "the reality is masked by those human processes which judge and deduce the phenomenon in consciousness prior to a full understanding of the structure of meaning it expresses." In other words, social reality is a protrusion of human imagination. For example, human nature is different from animals or physical objects, and it immediately narrates the gist of underlying phenomenon, situation or event. The ability to obtrude something shaped the world within a specified frame of reference in terms of different experiences and perceptions (Gill and Johnson, 2002). Figure 1 shows these two extremes in an objective-subjective continuum. As shown, the 'objectivist' supports an epistemological standpoint of the positivism; whereas the 'subjectivist' favors phenomenological epistemology (Morgan and Smircich, 1980). 
Figure 1. Objective-Subjective Continuum - Morgan and Simircich (1980)

\section{EPISTEMOLGY}

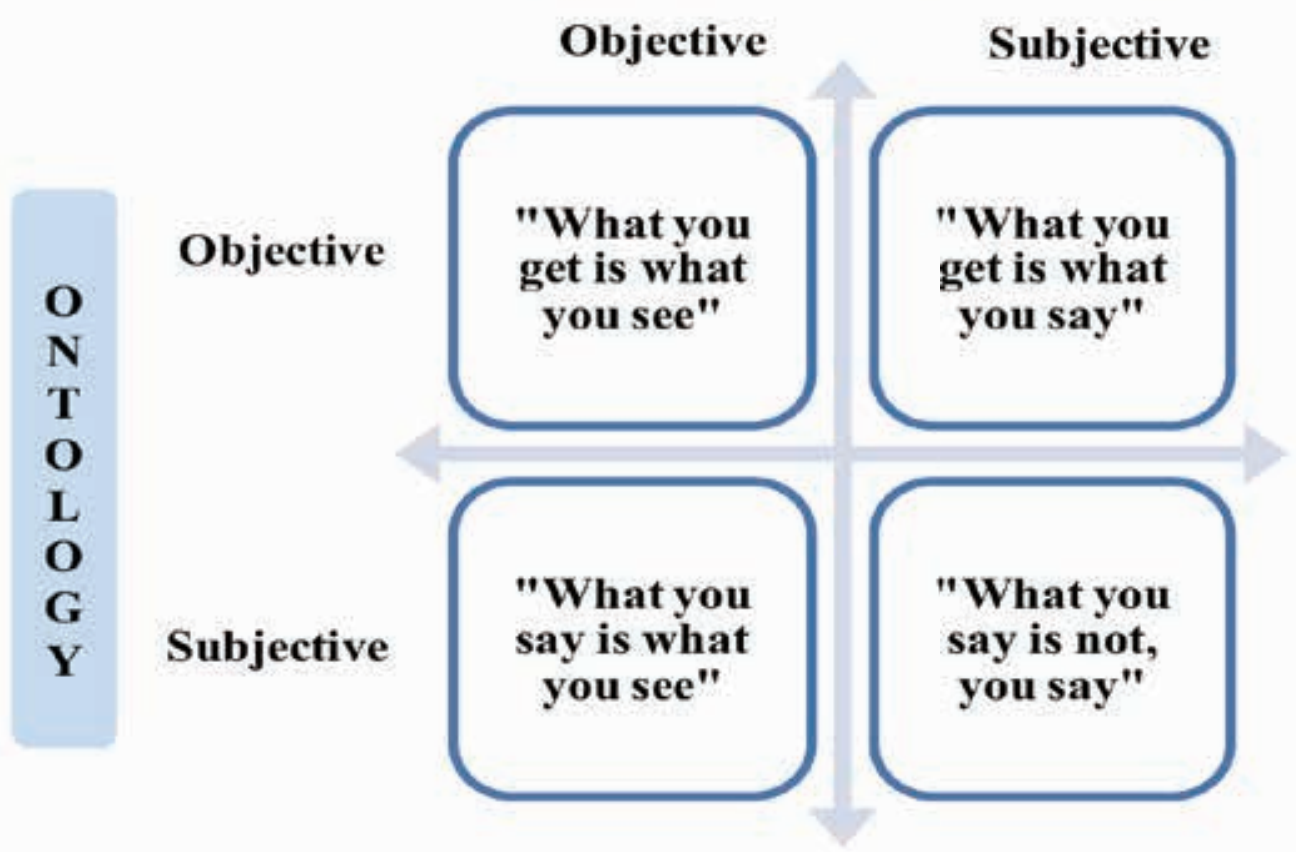

Issues of Epistemological Assumptions

On the other hand, epistemology is a branch of philosophy that is concerned with the study of knowledge (Collis and Hussey, 2009). Epistemology claims about 'what can be known', 'what exists may be known' and 'how and what it is possible to know' (Chia, 2003 and Blaikie, 2007). The adequacy and nature of knowledge requires that there is a specific way which informs us how to seek answers to the research question which is assumed to be true and show the reality. The way we see truth and reality reflects an epistemological position. For example, an epistemological position can be described as objectivists and constructivists. An objectivists hold the truth that is objective, and it is out there irrespective of our awareness. In contrast, constructivists hold that there is no any objective truth, it is to be constructed by the people with their interaction with this world (Crotty, 1998). Maynard quoted that "epistemology is concerned with providing a philosophical grounding for deciding what kind of knowledge is possible and how can we ensure that they are both adequate and legitimate." (Maynard, 1994, p.10)

\section{Research assumptions, question, method and approach}

Epistemology is also described in relation to ontology (Bhattacherjee, 2012). The term ontology as a philosophical perspective in social sciences claims about 'what is reality', 'what exists', 'what units make it up', and 'what it looks like' (Creswell, 2013, p.19). Laughlin (1995) posits that human behavior is also part of ontology. Thus, the role of the researcher in finding that reality is related to human behavior assumptions. 
Table 1: Research Assumption, Question, Method and Approach

Ontological Assumption: Culture and its impact on knowledge creation and the application of the SECI model will enrich the insights of an organisation into their knowledge creation and the processes involved in it (Haag et al., 2010).

Epistemological Assumption: Epistemologically can be assumed that different organisation culture values may have an impact on the employees' knowledge creation process.

Mixed Research Question: How does organisational culture affect knowledge creation process?

\begin{tabular}{lll}
\hline $\begin{array}{l}\text { Research Paradigm/Methods } \\
\text { i) Positivism (Quantitative) }\end{array}$ & ii) Post-Positivism (Qualitative) & iii) Pragmatism (Mixed) \\
\hline $\begin{array}{lll}\text { Research Approach } & \text { ii) Action Research } & \text { iii) Experiment } \\
\text { i) Ethnographic } & \text { v) Survey } & \\
\text { iv) Case Study } & \text { i) }\end{array}$
\end{tabular}

Therefore, it is not meaningless to say that, ontology is an assumption about the reality with respect to the people, culture and the world (Eriksson and Kovalainen, 2008). In contrast, an epistemology provides answers to the questions: what is the relationship between the researcher and that researched'? (Creswell, 2013, p.13). In other words, the knowledge that produced from epistemology termed as propositional knowledge. The 'propositional' knowledge is a valid knowledge which can be articulated in a declarative sense that may have ability to explain state of affair. For any research, ontological and epistemological assumptions make up a paradigm (Mack, 2010). The ontological assumption makes the researcher's mind for epistemological assumptions, which further make a choice of research methodology. For example, the ontological position is based on the assumption that culture may be a factor in knowledge creation through the application of the knowledge creation process (Haag et al., 2010).

However, the researcher assumed this standpoint at the epistemological level and posits that the different organisational culture values may have an impact on knowledge creation process. Literally, the researcher holds that the social actors play an accompanying role in attaining and interpreting language, slogans, codes and labels in order to understand and recognize reality (Morgan and Smircich, 1980). The role of human beings in gaining knowledge is obligatory as we cannot separate the knower and the known out from the discourse of social reality (Morgan and Smircich, 1980). In a similar vein, knowledge subsists in the reality of the world where human beings identify the nature of relationships among social phenomena (Johnson and Onwuegbuzie, 2004). Therefore, in spite of following any one of the philosophical standpoints within objectivism-subjectivism continuum, the researcher can hold a balanced position in his ontological and epistemoogical assumption. Hence, it is neither solely positivist (objective) nor interpretivist (subjective). For that reason, the researcher may be required to follow any mixed position that not only deals with the objective social world, but also provides some subjective implications so that we can infer and interrelate with the social world.

Methodologically, an important relationship between organisational culture and the knowledge creation process intends to establish on the basis of both the objective knowledge that can be gathered and measured empirically through the quantitative method and the subjective meaning of social action through a different logic of a research procedure. The researcher 
intended to take a balanced philosophical standpoint as it not only substantiates the reality and significance of the natural or physical world but it also acknowledges the importance of the social and psychological world. The complex nature of the research problem in the illustration mentioned above, however, require intense investigation.

For this purpose, the researcher can collect data from two different sources. For instance, the bank managers and employees provided information to investigate the causal relationship between organisational culture and knowledge creation. Thus, information about policy consideration on knowledge management initiatives can be obtained from senior managers and HR heads of the banks. For data collection, the researcher is required to follow mixed methodological position that not only deals with the objective social world, but it also provides the researcher with some subjective implications so that the researcher can infer and interrelate with the social world. However, while evaluating different methodological approaches, the researcher tends to choose both quantitative (positivism) and qualitative (post-positivism) paradigm in a single study.

\section{Positivism and post-positivist paradigm views in a mixed-methods}

Positivism is a tenant of positive epistemology. According to Morgan and Smircich (1980), the researchers with a positivist point of view do not hold the knowledge, but they can only observe and measure knowledge. In addition, the positivism is a philosophical approach of a scientific inquiry that presumes objective knowledge gathered from experience, direct observation and can be measured empirically through quantitative methods such as, survey and experiment (Hatch, 2012). The positivism (also known as scientific paradigm) usually accepts data based on scientific evidence and breaks down this data to isolate elements (Guthrie, 2010). Additionally, strict rules and procedures in a positivism may be useful to yield quantifiable data that will lead to either accept or reject the hypothesis (Robson, 2002). For instance, "positivists generate hypotheses from theory that can further be tested numerically that will thereby allow justifications of laws to be assessed." (Bryman, 2004, p.12)

In contrast, phenomenological (or normative, interpretive) epistemology is based on the subjective ontological assumption. The predecessors of subjective ontological and phenomenology epistemological assumptions usually adopt the qualitative methodological approach (Collis and Hussey, 2003; Bryman, 2004). Specifically, it differentiates the social science and natural science in terms of subjective norms and procedures. According to Morgan and Smircich (1980), phenomenologist emphasize the process through which human beings corroborate their subjective experience and consciousness. Unlike positivism, phenomenology provides the subjective implication with a different logic of the research procedure (Bryman, 2004). The 'objective-ontological' and 'positive-epistemological' assumptions follow a quantitative research methodology. A quantitative method tends to apply quantifications in the data collection and data analysis. It aims to employ deductive approach to look intently at the relationship between theory and research. It also facilitates theory testing and developing hypotheses. However, a quantitative approach requires specific samples and large data in order to make generalizations (Collis and Hussey, 2009). Furthermore, quantitative researchers use a positivist claim for developing knowledge through theory testing by employing strategies of inquiry such as experiments or surveys. For this purpose, quantitative data can be collected by predetermined instruments (Creswell, 2013). 
On the other hand, a qualitative design is generally associated with the post-positivist philosophical level usually employs to generate theories from induction (or 'inductive logic') (Bryman, 2004). Post-positivism is one of the most common philosophical approaches that consider knowledge as a subjective phenomenon (Guthrie, 2010). Post-positivists assert that human knowledge is based on human hypothesis. Therefore, human knowledge is entirely hypothetical; whereas, confirmation of hypotheses is acceptable through subjective interpretation than by theory testing using quantitative models (Bryman, 2004; Guthrie, 2010). Moreover, as a model of scientific enquiry, post-positivism depicts a meta-theoretical stance that critiques and amends positivism. Therefore, critical realists endeavor to resolve criticisms about positivism. It also recognizes that all observations are imperfect, and all theories may be reversible because the researcher can get closer to the truth through theories, but it may not obtain the real truth (Collis and Hussey, 2009).

\section{Philosophical and methodological contradiction in a mixed-methods}

It has long been debated that the methodology used in a physical sciences might not fit in social sciences due to a number of reasons (Onwuegbuzie, 2002). For instance, the qualitative and quantitative methodologies stand on different philosophical assumptions thereby incompatible with each other (Smith and Heshusius, 1986). However, the concept of using both qualitative and quantitative methods in a single study derived from Campbell and Fiske (1959, p.81) who wrote that "in order to measure the relative contributions of trait and method variance, multiple traits, as well as multiple methods, must be utilized in the validation process." In this connection, the concept of methodological triangulation (or mixing of a two datasets) is most common as it facilitates the validation of data through cross-verification of data sets from more than two sources (e.g. interviews, observations, questionnaires and documents) (Johnson and Onwuegbuzie, 2004; Bogdan and Biklen, 2006; Denzin and Giardina, 2006; Onwuegbuzie and Leech, 2007).

However, in terms of philosophical underpinning, the conflicting and contradictory views of both positivist philosophies (quantitative design) and post-positivist philosophies (qualitative design) frequently utilized in conjunction (Jogulu and Pansiri, 2011). Therefore, mixed-methods research usually employs a pragmatic approach as a system of philosophy (Johnson and Onwuegbuzie, 2004). For instance, researchers normally build knowledge on pragmatic grounds (Creswell, 2013). It offers a workable solution either philosophically or methodologically when the main theory exists on a quantitative and qualitative research paradigm. Furthermore, pragmatism as a system of philosophy extends the result of an oriented technique of enquiry with the required logic of justification thus providing a suitable methodological fit amid different (or mixed) paradigms (Johnson and Onwuegbuzie, 2004). Pragmatism, as a philosophical approach, views knowledge as an indispensable reality or an intimate experience (Johnson and Onwuegbuzie, 2004). Pragmatists believe that existing truth, implication, and the boundaries of knowledge are impermanent thus knowledge can be changed, modified or altered with or without research over time (Johnson and Onwuegbuzie, 2004). 


\section{Mixed-methods validity in a management research}

Although, a massive amount of empirical research has been carried out on the knowledge management research agenda, there is "no identifiable research methodology" that can serve the purpose (Wallace et al., 2010, p.5). For example, a 60\% of the knowledge management researchers employed research methodologies and were typically derived from the social sciences research (Wallace et al., 2010). Due to no identifiable research methods and over-dependence on the research methods used in the social sciences, the researcher can use the research method on the basis of three yardsticks. First, a research method that better served the purpose. Second, a research method widely used in management sciences research. Third, a research method widely used in knowledge management research. For example, research methods that have been widely used in management studies can be categorized into three transitions; i.e. earlier, later and most recent (Karami et al., 2006). Karami et al. (2006) reported that the research methods used in management studies were typically based on positivism as a philosophical approach of a scientific enquiry with an early emphasis on case studies; a later emphasis on empirical methods; and a more recent emphasis on the qualitative and phenomenological methods.

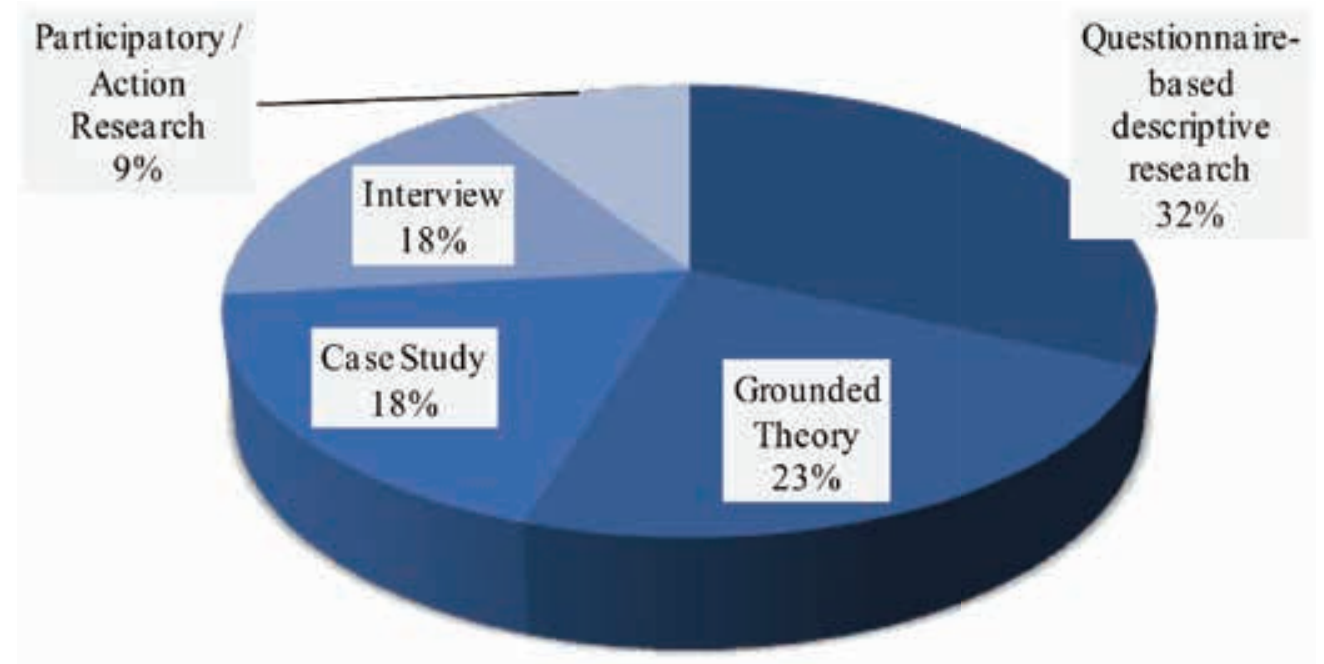

Figure 2. Research Methods Used in Management Research - Karami et al (2006)

Out of 217 articles published in the leading management journals, a total of 32\% (69) were questionnaire-based descriptive research, 23\% (50) used the grounded theory approach, $18 \%$ (40) employed the case study method, $18 \%$ (38) conducted interviews and $09 \%$ (20) were based on action research. In the case of knowledge management research, out of 630 articles published during 2006 and 2008 in the most recognized knowledge management journals, $28 \%$ (175) used no identifiable research methodology while $72 \%$ (455) used provisionally identifiable research methods (see Figure 2). As shown in the breakdown of the provisionally identifiable research methods, $26.8 \%$ were qualitative case studies, $16.9 \%$ used questionnaire survey, $11.9 \%$ based on interviews, $1.8 \%$ consists of field studies and $5.3 \%$ of the articles used miscellaneous social science research methods (Wallace et al., 2010). In addition, the suitability of the research method may also be evaluated through the relevancy between the research question and the research method. 


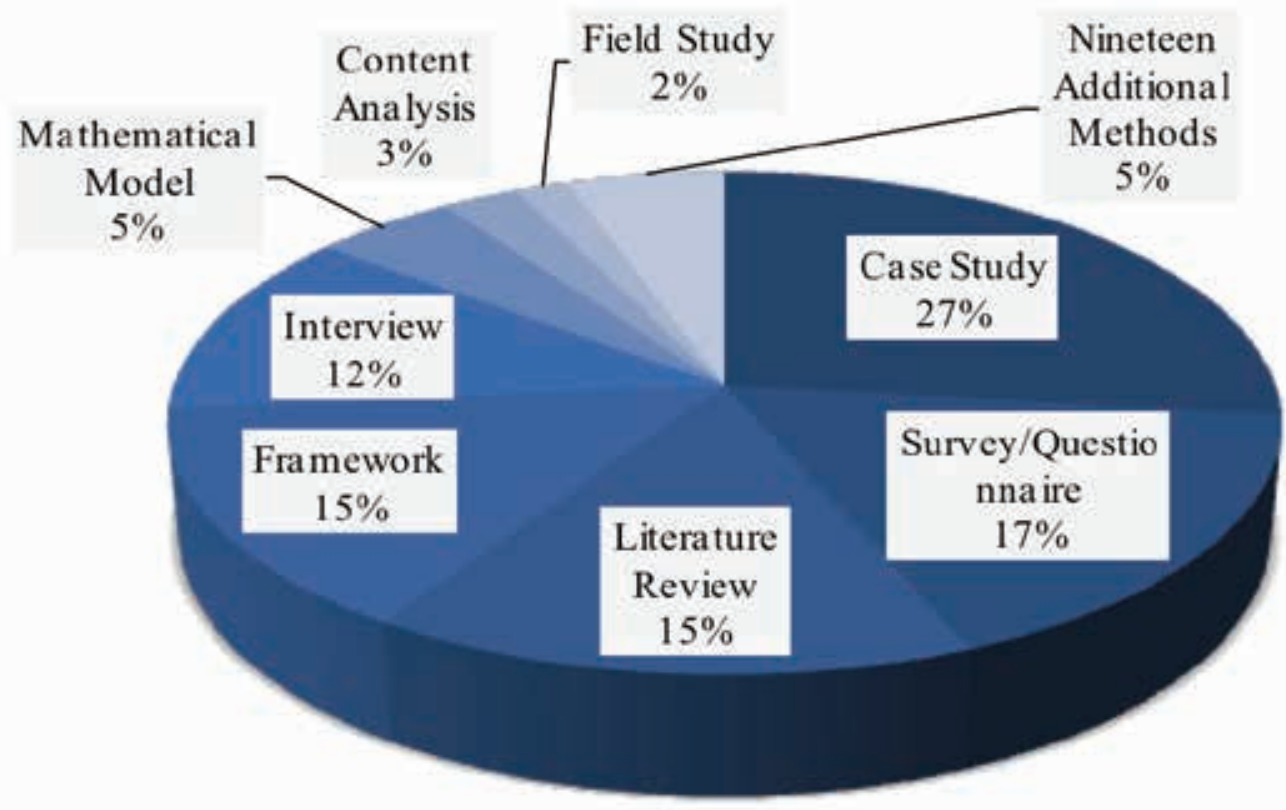

Figure 3. Research Methods in KM Research - Wallace et al (2010)

The multifaceted nature of the research problem and involvement of many independent and dependent variables require integration of quantitative and qualitative dataset into a single investigation to address the underlying problem in more detail. Hence, the complex research questions (see Table 1) may better be addressed through a mixed quantitative (objectivist $\mathbb{Q}$

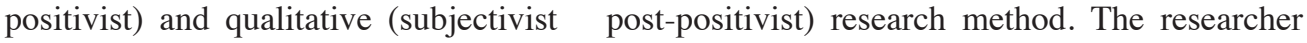
believes that no research paradigm is better than another as each is suited to its particular purpose. In the case of research methods used in the organizational culture and knowledge creation research, the researcher browsed through the relevant knowledge management journals and extracted twenty relevant publications. It revealed that, out of 20 articles, 30\% were case studies, $25 \%$ were quantitative, $20 \%$ were qualitative, $10 \%$ were mixed-methods and $15 \%$ were conceptual papers. Also, the researcher might not get rid of the likely influence of top level management and HR managers that may sabotage the research process. For example, in organizational domain, research process carry out in an uncontrolled and context specific environment. 


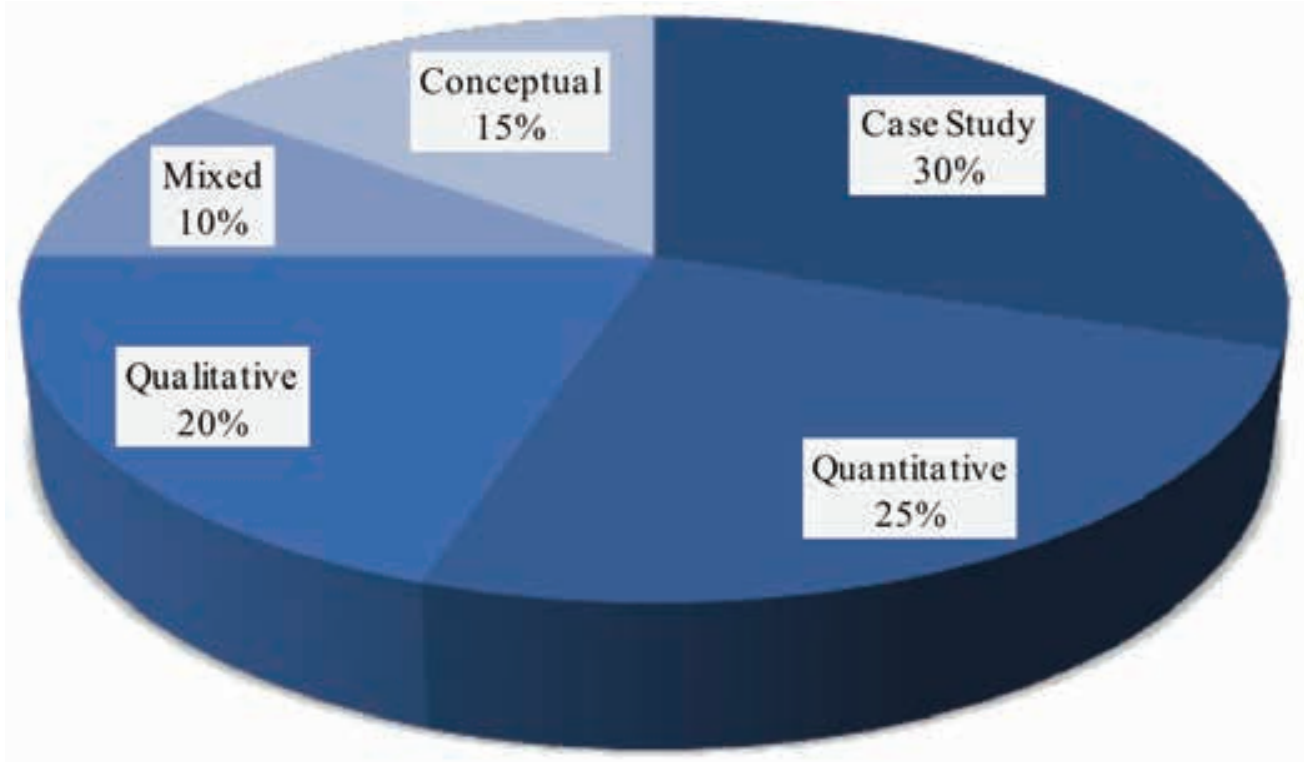

Figure 4. Research Methods in Organizational Culture and Knowledge Creation

In such a situation, the study requires a qualitative research method or post-positivism as a system of philosophy in order to address 'what' and 'how' questions and unfold the exploratory nature of the underlying problem (Yin, 2003). For that purpose, a qualitative portion needs to measure qualitatively by interviewing from top level management and HR managers in order to tap their perceptions about the policy framework. In addition, a quantitative study might not be suitable for exploring the research phenomenon because there is a lack of established models that can explicitly measure the relationship between organisational culture and knowledge creation. Therefore, a qualitative study coupled with quantitative study used to recognize the relationships among organisational culture and knowledge creation process to build more concrete evidence. More specifically, a mixed-methods research has been utilized as the best method that combines quantitative and qualitative methods to investigate organisational culture and knowledge creation. More to the point, a mixed-methods research provides a best fit to address the research question than individual method.

\section{Methodological aspects of mixed-methods research design}

The process of a research design consists of 'gathering', 'assessing', 'inferring', and 'reporting' data (Creswell and Plano Clark, 2007). According to the Yin, research design is a "logical illustration that relates the data to be collected and the conclusions to be drawn to the initial questions of a study." (Yin, 2003, p.6).

In a mixed-method study, literature indicates a variety of design options depending upon the decision points such as; interaction, priority, timing, and mixing (Creswell, 2013). For example, Tashakkori and Teddlie (1998) reported three types: i) equivalent status design; ii) dominant/less dominant design; iii) multilevel design. Likewise, Creswell (2013) outlined six basic designs namely: i) convergent parallel design; ii) explanatory sequential design; iii) explorato- 
ry sequential design; iv) embedded design; v) transformative design; vi) multiphase design. However, before choosing a suitable mixed-method design, the researcher ponders to be consistent and clear in terms of four key decisions such as; i) the degree of an interface; ii) priority; iii) implementation; iv) mixing (Creswell, 2013). The decision of adopting the level of interaction between the quantitative and qualitative threads may either be independent or interactive (Greene, 2007). An independent level of interaction executes distinctively. For example, research questions, data collection, and data analysis of both threads depends on each other. However, the results of both strands can only be mixed while drawing conclusions at the end of the study.

Table 2. Key Decisions in Mixed-Methods

\begin{tabular}{llll}
\hline Level of Interaction & Priority & Timing & Mixing \\
\hline Independent & Qualitative & Concurrent & In Interpretation \\
Interactive & Quantitative & Sequential & In Data Analysis \\
& Equal & Multiphase Combination & In Data Collection \\
\hline
\end{tabular}

In contrast, the level of interaction in an interactive design permit direct interaction between the quantitative and qualitative strands so that the both can be mixed before the final interpretation. Also, an interactive design allows researchers to mix the results from one type of strand into the other type of strand so that the different data sets can be analyzed together (Creswell, 2013). In the third decision of priority, researcher ought to weigh the prerequisites of quantitative and qualitative methods before addressing the research questions (Creswell et al., 2003). For instance, the decision of fixing priority i.e. implicitly or explicitly based either upon the research question or the researcher prudence of solving the problem.

\footnotetext{
1 - The convergent parallel design occurs when the researcher uses concurrent timing to implement the quantitative and qualitative strands during the same phase of the research process, prioritizes the methods equally, and keeps the strands independent during analysis and then mixes the results during the overall interpretation. The explanatory sequential design starts with the collection and analysis of quantitative data, which has the priority for addressing the study's questions. This first phase is followed by the subsequent collection and analysis of qualitative data. The second, qualitative phase of the study is designed so that it follows from the results of the first, quantitative phase. The researcher interprets how the qualitative results help to explain the initial quantitative results. The exploratory sequential design begins with and prioritizes the collection and analysis of qualitative data in the first phase. Building from the exploratory results, the researcher conducts a second, quantitative phase to test or generalize the initial findings. The researcher then interprets how the quantitative results build on the initial qualitative results. The embedded design occurs when the researcher collects and analyses both quantitative and qualitative data within a traditional quantitative or qualitative design. In an embedded design, the researcher may add a qualitative strand within a quantitative design, such as an experiment, or add a quantitative strand supplemental strand is added to enhance the overall design in some way. The transformative design is a mixed methods design that the researcher shapes within a transformative theoretical framework. All other decisions (interaction, priority, timing and mixing) are made within the context of the trmative theoretical framework. All other decisions (interaction, priority, timing, and mixing) are made within the context of the transformative framework. The multiphase combines both sequential an concurrent strands over a period of time that the researcher implements within a program of study addressing an overall program objective. This approach is often used in program evaluation where quantitative and qualitative approaches are used over time to
support the development, adaptation, and evaluation of specific programs (Creswell, 2013 p. 71-72). 


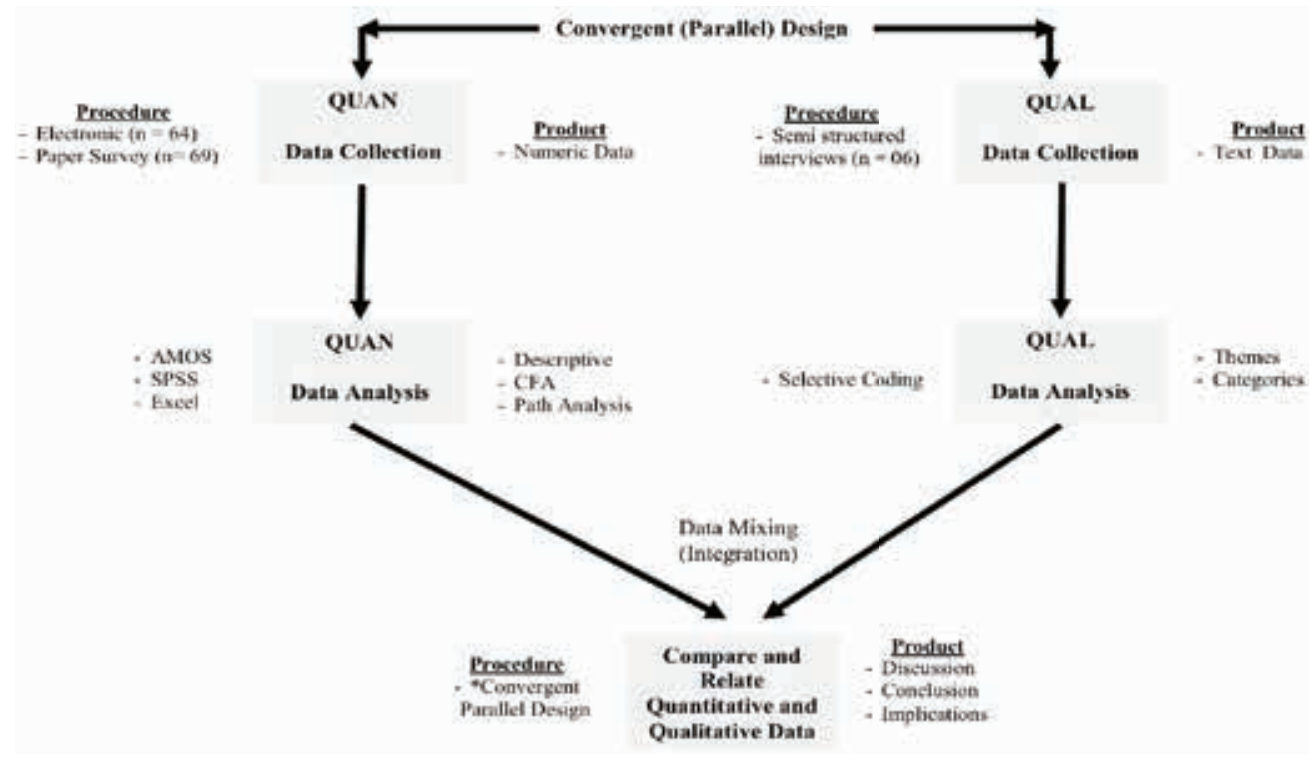

Figure 5. Mixed-Method Research Design - Creswell (2013)

In this connection, the researcher's philosophical underpinning and research aims and objectives play a significant role in selecting data collection and analysis processes (Creswell, 2013). Arguably, the decision of imparting priority was made on the basis of research aims and objectives. The purpose of the quantitative strand is to categorize 'predictive power' of the hypothesized relationship. However, qualitative method used to collect textual data through semi-structured interviews. Purpose of qualitative strand is to clarify why certain internal and external factors, tested in the quantitative survey, may be potential significant predictors of the employees' knowledge creation phenomenon in banking organizations.

The timing refers to the implementation decision in which quantitative and qualitative data to be collected and analyzed (Creswell, 2013). Timing relates to the preference of the researcher in which he/she implement quantitative and qualitative studies either concurrently, sequentially or multiphase combination (Ivankova et al., 2006; Creswell, 2013). For example, concurrent (or parallel) design allows researchers to implement qualitative and quantitative strand during a single phase approximately at the same time. In contrast, sequential design or timing implement in two distinct phases in which data collection and analysis of each strand carried out individually in two phases. However, multiphase timing is a combination of both 'concurrent' and 'sequential' elements within mixed-methods study. For example, this study has opted concurrent mixed-method design due to several theoretical and practical concerns. Specifically, the concurrent (or parallel) design allows researchers to collect both type of data in a shorter period that equally support the research questions during data analysis (Creswell, 2013). The last important decision relates to data integration of two data sets. According to Morse and Niehaus (2009), the two datasets can be mixed at the point of interface. For this, the both data sets can be mixed in such a way that both strands do not overlook important implication thus it extends the synergic effect in findings and analysis (Woolley, 2009). 


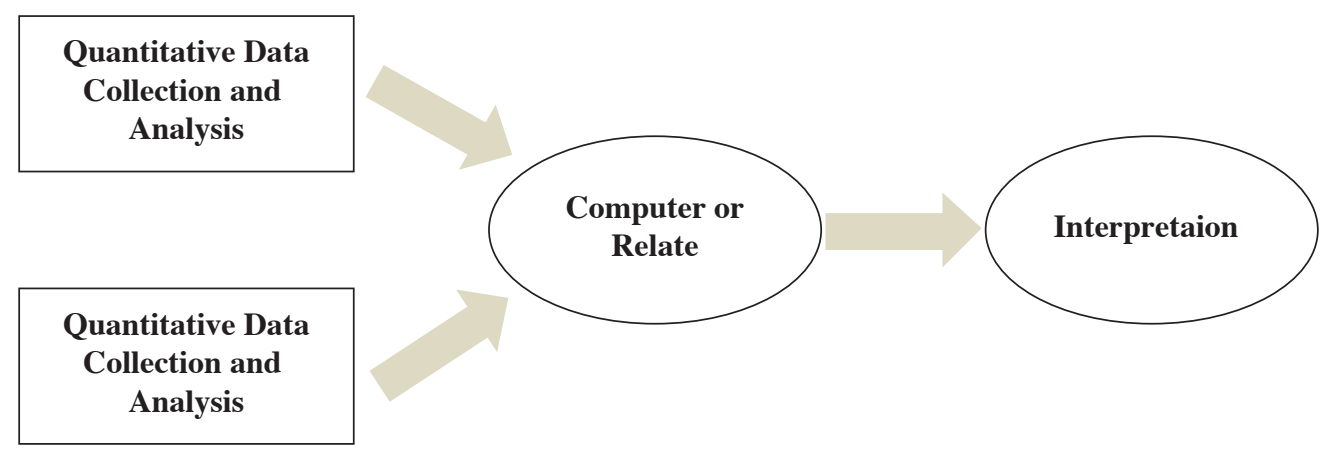

Figure 6: The Convergent Parallel Design - Creswell (2013)

In case of example illustrated as above, the researcher keeps the two strands (see Figure 6) independent and the both types of data strands execute distinctively. The quantitative strand collected through electronic and paper based survey. Whereas; the semi-structured interviews conducted for the qualitative data. In quantitative analysis, the adequacy of the hypothesized relationship can be assessed through the structural equation modelling (SEM) with the help of statistical package IBM AMOS v19. Whereas; the researchers' can use Nvivo for managing and analyzing qualitative data collected during semi-structured interviews. The results of the quantitative study and results of a qualitative study can be reported separately. However, the mixing of the two strands (datasets) occurs in the final step during discussion and conclusion stage.

More specifically, data mixing in the final stage of study allows researchers in drawing valid inferences that reflect the apparent picture of two data strands, such as by relating or combining the findings (Creswell, 2013). For instance, the analysis of quantitative and qualitative data seeks to bring the data together in order to answer the set of research questions unable to be effectively answered by the individual methods. The mixed-method design keep the two strands independent. The mixing of two strands occur at the interpretation stage. However, the both data findings finally relate and compare at the last stage using "convergent parallel design' (see Figure 6). Specifically, the purpose of convergent design is to attain a complementary data for in depth results (Greene and Caracelli, 1997). For example, the use of both numerical and non-numerical data, however, complements each other in order to reach at valid conclusion. The use of this design allows researchers to triangulate the quantitative design (e.g. large sample size, numbers, trends, and generalization) with qualitative design (e.g. small sample size, text, descriptive details, and in depth) through relating and comparing both results for confirmation purpose (Creswell, 2013). Hence, the final decision of choosing a convergent design also made due to close semblance between design and study's objectives.

\section{CONCLUSION}

The selection of the research method is not subject to the merits and demerits of that method. Likewise, there is no such yardstick that could corroborate the suitability of any of the research methods in terms of their strengths and limitations. Therefore, the researcher needs to be persistent, devoted and a lot of rigor in order to apply mixed-methods for valid and reliable findings. Also, final selection of research process is inherently associated to the research

44 Jan-June $2017 \quad$ Volume $1 \quad$ Number $15 \quad$ JISR-MSSE


problem and the way through which it seeks answers. The mixed-method research currently being accepted as a 'new research paradigm' with an intention not only to reinstate the prevailing paradigms but to mitigate the weaknesses of individual research approaches (Creswell, 2013). In recent management studies, the use of this research design generally prefer to ratify the reliability and validity of the overall research findings and to 'bridge the gap' between research and theory (Creswell and Plano Clark, 2007). More specifically, the efficient use of mixed-method technique provides more extensive results from which accurate and credible inference can be drawn. However, in a deliberate attempt of using mixed-methods design, the researcher ought to consider the possible limitations of the final research process thus also taken certain measures to rationalize the potential limitations of mixed-methods design including lack of representativeness and validity of statistical findings. For example, the qualitative data gathered from a small number of participants cannot be generalized to the wider community. Methodologically, it does not invalidate the overall findings, but the researcher can never generalize these findings with certainty to other persons, places or times that are more 'proximally similar'. Therefore, the mixed design can be used to generate an 'external validity' in the findings and provided more opportunities to make suggestions for future endeavors. All these aspects, however, effect the decision to employ a mixed-methods which seem to be a useful tool in terms of diversity, integrity and comprehensiveness by way of gathering and measuring an objective knowledge empirically through a quantitative method and subjective interpretations in relation to social phenomena that through a different logic of research procedure of a qualitative method.

\section{REFERENCES}

Bryman, A. (2004). Qualitative research on leadership: A critical but appreciative review. The Leadership Quarterly, , 15, 729-769.

Bryman, A. (2005). Quantitative Data Analysis with SPSS Release 12 and 13: A Guide for Social Scientists. Routledge.

Burnette, J.L. and Williams, L.J. (2005). Structural equation modeling (SEM): An introduction to basic techniques and advanced issues. Research in organizations: Foundations and methods of inquiry, 143-160.

Bogdan, R. 86. Biklen. S. K (2006). Qualitative research in education: An introduction to theory and methods London, Allyn \& Bacon,

Bryman, A. (2007). The research question in social research: what is its role? International Journal of Social Research Methodology, 10, 5-20.

Blaikie, N. (2007). Approaches to social enquiry: Advancing knowledge. Polity.

Bhattacherjee, A. (2012). Social Science Research: Principles. Methods and Practices $2^{\text {nd }}$ ed., Global Text Project,

Campbell, D.T. and Fiske, D.W. (1959). Convergent and discriminant validation by the multitrait-multimethod matrix. Psychological bulletin, 56, 81.

\begin{tabular}{llll}
\hline JISR-MSSE & Volume 1 & Number 15 & Jan-June 2017 \\
\hline
\end{tabular}


Crotty, M. (1998). The foundations of social research: Meaning and perspective in the research process. Sage.

Collins, J. and Hussey, R. (2003). Business research. Hampshire, UK: Palgrave Macmillan,

Chia, R. (2003). Ontology: organization as "world-making". Debating organization: point-counterpoint in organization studies, pp.98-113.

Collis, J., Hussey, R., Crowther, D., Lancaster, G., Saunders, M., Lewis, P., Thornhill, A., Bryman, A., Bell, E. and Gill, J. (2003). Business research methods.

Creswell, J.W., Plano Clark, V.L., Gutmann, M.L. and Hanson, W.E. (2003). Advanced mixed methods research designs. Handbook of mixed methods in social and behavioral research, 209-240.

Cassell, C., Symon, G., Buehring, A. and Johnson, P. (2006). The role and status of qualitative methods in management research: an empirical account. Management Decision, 44, 290-303.

Creswell, J.W. and Plano Clark, V. (2007). Choosing a mixed methods design. Designing and conducting mixed methods research, 58-88.

Corbin, J. and Strauss, A. (2008). Basics of qualitative research: Techniques and procedures for developing grounded theory. Sage.

Creswell, J.W. (2009). Editorial: Mapping the field of mixed methods research. Journal of Mixed Methods Research, 3, 95-108.

Collis, J. and Hussey, R. (2009). Business research: A practical guide for undergraduate and postgraduate students. Palgrave Macmillan.

Creswell, J.W. (2013). Research design: Qualitative, quantitative, and mixed methods approaches. Sage Publications, Incorporated.

De Waal, C. (2001). On Peirce. Wadsworth Publishing Company.

Denzin, N.K. and Giardina, M.D. (2006). Qualitative inquiry and the conservative challenge. Left Coast Press.

Denzin, N.K. and Lincoln, Y.S. (2011). The SAGE handbook of qualitative research. Sage.

Eriksson, P. and Kovalainen, A. (2008). Qualitative methods in business research. Sage.

Easterby-Smith, M. and Richard-Jackson, P.R. Lowe, Andy. (2008). Management Research third edition. London. Sage, 
Greene, J.C., Caracelli, V.J. and Graham, W.F. (1989). Toward a conceptual framework for mixed-method evaluation designs. Educational evaluation and policy analysis, $11,255-274$.

Gill, J. and Johnson, P. (2002). Research methods for managers. Sage.

Guthrie, G. (2010). Basic research methods: An entry to social science research. Sage Publications.

Grafton, J., Lillis, A.M. and Mahama, H. (2011). Mixed methods research in accounting. Qualitative Research in Accounting \& Management, 8, 5-21.

Hughes, Jim and Jones, Steven eds. (2003). Reflections on the use of grounded theory in interpretive information systems research. ECIS.

Harrington, D. (2008). Confirmatory factor analysis. Oxford University Press.

Haag, M., Duan, Y. and Mathews, B. (2010). The impact of culture on the application of the SECI model. Cultural Implications of Knowledge Sharing, Management and Transfer: Identifying Competitive Advantage, 26-47.

Hatch, M.J. (2012). Organization theory: modern, symbolic and postmodern perspectives. Oxford university press.

Ivankova, N.V., Creswell, J.W. and Stick, S.L. (2006). Using mixed-methods sequential explanatory design: From theory to practice. Field Methods, 18, 3-20.

Johnson, R.B. and Onwuegbuzie, A.J. (2004). Mixed methods research: A research paradigm whose time has come. Educational researcher, 33, 14-26.

Johnson, R.B., Onwuegbuzie, A.J. and Turner, L.A. (2007). Toward a definition of mixed methods research. Journal of mixed methods research, 1, 112-133.

Jogulu, U.D. and Pansiri, J. (2011). Mixed methods: a research design for management doctoral dissertations. Management research review, 34, 687-701.

Kaplan, B. and Duchon, D. (1988). Combining qualitative and quantitative methods in information systems research: a case study. MIS quarterly, 571-586

Karami, A., Rowley, J. and Analoui, F. (2006). Research and knowledge building in management studies: an analysis of methodological preferences. International Journal, $23,43-52$

Kline, R.B. (2011). Principles and practice of structural equation modeling. Guilford press. 
Laughlin, R. (1995). Empirical research in accounting: alternative approaches and a case for "middle-range" thinking. Accounting, Auditing \& Accountability Journal, 8, 63-87.

Morgan, G. and Smircich, L. (1980). The case for qualitative research. Academy of management review, 5, 491-500.

Morse, J.M. (1994). Critical issues in qualitative research methods. Sage.

Maynard, M. (1994). Methods, practice and epistemology: The debate about feminism and research. Researching women's lives from a feminist perspective, 10, 26.

Modell, S. (2010). Bridging the paradigm divide in management accounting research: the role of mixed methods approaches. Management Accounting Research, 21, 124-129.

Mack, L. (2010). The philosophical underpinnings of educational research. 5-11.

Nonaka, I. (1994). A dynamic theory of organizational knowledge creation. Organization science, 5, 14-37.

Onwuegbuzie, A.J. (2002). Why Can't We All Get Along Towards A Frame Work for Unifying Research

Paradigms. Education-Indianapolis Then Chula Vista, 122, 518-530.

Onwuegbuzie, A.J. and Leech, N.L. (2006). Linking research questions to mixed methods data analysis procedures. The Qualitative Report, 11, 474-498.

Pansiri, J. (2005). Pragmatism: a methodological approach to researching strategic alliances in tourism. Tourism and Hospitality Planning \& Development, 2, 191-206.

Pansiri, J. (2008). The effects of characteristics of partners on strategic alliance performance in the SME dominated travel sector. Tourism Management, 29, 101-115.

Robson, C. (2002). Real world research: A resource for social scientists and practitioner-researchers. Blackwell Oxford.

Smith, J.K. and Heshusius, L. (1986). Closing down the conversation: The end of the quantitative-qualitative debate among educational inquirers. Educational researcher, 15, 4-12.

Tashakkori, A. and Teddlie, C. (1998). Mixed methodology: Combining qualitative and quantitative approaches. SAGE Publications, Incorporated.

Tashakkori, A. and Creswell, J.W. (2007). Editorial: The new era of mixed methods. Journal of mixed methods research, 1, 3-7. 
Tashakkori, A. and Teddlie, C. (2010). Sage handbook of mixed methods in social \& behavioral research. Sage.

Woolley, C.M. (2009). Meeting the mixed methods challenge of integration in a sociological study of structure and agency. Journal of Mixed Methods Research, 3, 7-25.

Wallace, D.P., Fleet, C.V. and Downs, L.J. (2010). The use of research methodologies in the knowledge management literature. Proceedings of the American Society for Information Science and Technology, 47, 1-7.

Yin, R.K. (2003). Case study research: Design and methods. Sage 
